\title{
Mixed Convection and Radiative Heat Transfer of MHD Casson Fluid Flow by a Permeable Stretching Sheet with Variable Thermal Conductivity and Lying in Porous Medium
}

\author{
Hassan Waqas ${ }^{1}$, Sajjad Hussain ${ }^{1 *}$, Rabia Naseem ${ }^{1}$, Amna Mariam ${ }^{1}$ \\ and Shamila Khalid ${ }^{1}$ \\ ${ }^{1}$ Department of Mathematics, Govt. College University Faisal Abad (Layyah Campus), Pakistan.
}

Authors' contributions

This work was carried out in collaboration between all authors. Author HW managed the analysis of the study, performed the statistical analysis. Author SH designed the study and managed the technical aspects. Author RN obtained the numerical solution of problem. Author AM wrote the first draft of the study. Author SK managed the literature review. All authors read and approved the final manuscript.

Article Information

DOI: $10.9734 / \mathrm{BJMCS} / 2017 / 33762$

Editor(s):

(1) Dr. M. Subhas Abel, Department of Mathematics, Gulbarga University, India.

Reviewers:

(1) Aleksandar Boričić, University of Niš, Serbia.

(2) Farhad Ali, City University of Science and Information Technology, Peshawar Pakistan. Complete Peer review History: http://www.sciencedomain.org/review-history/19614

\begin{abstract}
This work investigates the mixed convection radiative heat transfer of electrically conducting Casson fluids. The fluid flows past a permeable stretching sheet lying in the porous medium. The heat transfer involves variable thermal conductivity and convective boundary conditions. The formulation of the problem is primarily in the form of the non-linear partial differential equations. These governing equations are transformed to their ordinary differential form by employing similarity transformation. The resulting equations are then solved numerically by classical Rung-Kutta method. Computations have been made for some representative values of the pertinent parameters to elaborate the physical behavior of flow and thermal characteristics.
\end{abstract}

Keywords: Mixed convection; Casson fluids; porous medium; stretching sheet; similarity transformation.

*Corresponding author: E-mail: sajjadgut@gmail.com;

E-mail:syedhasanwaqas@hotmail.com; 


\section{Introduction}

The study of magneto hydrodynamic (MHD) flow of non-Newtonian fluids in a porous medium has attracted many researchers due to its application in the optimization of solidification processes of metals, alloys, the geothermal sources investigation and nuclear fuel debris treatment. Magneto hydrodynamics concepts are utilized by the engineers in the design of heat exchangers, pumps, thermal protection, in space vehicle propulsion, control and re-entry, and in creating novel power-generating systems. The purification of molten metals from non-metallic inclusions through the application of magnetic field is another important feature of MHD. All such applications of MHD give rise to investigate the problems which involves the magneto hydrodynamic effects. The theoretical study of two dimensional non-Newtonian incompressible fluid flow over a surface with stretching or shrinking properties has taken the significant attention in the past few years due to its wide applications in engineering fields as well as in the industry. Some applications include the production of toothpaste, shampoo, custard solution, blood treatment, glass fiber production and design of the plastic films. The various non-Newtonian fluids are power-law fluids, micro polar fluids, viscoelastic fluids, Jeffrey fluid, Rivlin- Ericksen fluids, Casson fluids, Walter's liquid B fluids etc. Although various types of non-Newtonian fluid models are proposed to explain the different behavior, one of the most important types of non-Newtonian fluids is Casson fluid. The Casson fluid is a plastic fluid, which yields shear stress in constitutive equations. Some of the examples of Casson fluid model are jelly, soup, honey, tomato sauce, concentrated fruit juices, drilling operations, food processing, metallurgy, paints, coal in water, synthetic lubricants, manufacturing of pharmaceutical products, synovial fluids, sewage sludge and many others. Human blood is also considered as Casson fluid because of the presence of several substances like protein, fibrinogen and globin in aqueous base plasma in the blood. Human red blood cells form a chain like structure, known as aggregates or rouleaux. Casson [1] introduced this model to predict the flow behaviour of pigment oil suspensions of the printing ink type. Recently, Sulochana et al. [2] discussed the influence of non- linear thermal radiation on MHD 3-dimension Casson fluid flow with viscous dissipation. Nadeem et al. [3] examined the magnetohydrodynamic (MHD) boundary layer flow of a Casson fluid over an exponentially penetrable shrinking sheet. Raju et al. [4] studied the effects of heat and mass transfer on MHD Casson fluid flow past an exponentially permeable stretching sheet. Later on, several researchers studied Casson fluid pertaining to different flow situations. The unsteady boundary layer flow and heat transfer of a Casson fluid over a moving flat plate with a parallel free stream was studied by Mustafa et al. [5]. The exact solution for boundary layer flow of Casson fluid over a permeable stretching/shrinking sheet with and without external magnetic field was discussed by Bhattacharyya et al. [6-7]. The Casson fluid has an infinite viscosity at zero rate of shear and a yield stress below which no flow occurs and a zero velocity at an infinite shear rate [8-9]. Nadeem [10] has studied MHD flow of a Casson fluid over an exponentially/linearly shrinking sheet. Animasaun [11] has studied MHD dissipative Casson fluid flow with suction and nth order of chemical reaction. Nadeem [12] has discussed on Casson fluid past a linearly stretching sheet with convective boundary condition. Akbar [13] has studied Metachronal beating of cilia under the influence of Casson fluid and magnetic field. Akbar [14-15] has investigated the magnetic field effects on Eyring- Powell/Casson fluid flow toward a stretching sheet, asymmetric channel and Plumb Duct. Benazir et al. [16] have studied unsteady MHD Casson fluid flow over a vertical cone and flat plate with non-uniform heat source/sink.

Hassan et al. [17] studied chemical diffusion and radiative heat transfer effects on magnetohydrodynamics stagnation point flow of Casson fluid over a porous shrinking sheet. Recently, Hassan et al. and Nadeem et al. [18-19] investigated the unsteady magnetic hydrodynamic (MHD) stagnation point flow of Casson fluids with radiation. Nadeem et al. [20] presented the Atangana and Baleanu (AB) fractional derivative idea for the first time to study the free convection flow of a generalized Casson fluid due to the combined gradients of temperature and concentration. Nadeem et al. [21] worked on the Atangana and Baleanu (AB) in their recent work and introduced a new version of fractional derivatives which uses the generalized Mittag-Leffler function as the non-singular and non-local kernel and accepts all properties of fractional derivatives. Farhad et al. [22] considered the effects of magnetohydrodynamics on the blood flow when blood is represented as a Casson fluid, along with magnetic particles in a horizontal cylinder. 
The flow through porous media has a bearing in the progress of several applications, such as chemical reactors, geology, combustion, drying and liquid composite molding, and biological applications. Flow through a porous wall with convective acceleration was studied by Yamamoto and Yoshida [23]. The steady flow of a viscous fluid through a saturated porous medium of finite thickness, impermeable and thermally insulated bottom and the other side being stress free, at a constant temperature was studied by Mounuddin and Pattabhiramacharyulu [24]. Chamkha [25] investigated MHD free convection from a vertical plate embedded in a thermally stratified porous medium. MHD mixed convection from a vertical plate embedded in a porous medium with a convective boundary condition was investigated by Makinde and Aziz [26]. Makinde and Mhone [27] considered the temporal stability analysis for hydromagnetic flow in a channel filled with a saturated porous medium. The hydromagnetic mixed convection flow of an incompressible viscous electrically conducting fluid and mass transfer over a vertical porous plate with constant heat flux embedded in a porous medium is investigated by Makinde [28].

Hayat et al. [29] studied the effects of variable thermal conductivity on the mixed convection flow over a porous stretching surface for Newtonian fluids, without radiation. We made a comprehensive mathematical and computational analysis of this problem to examine the flow of the Casson fluids through porous medium and radiation.

\section{Mathematical Model}

Consider the mixed convective, steady, two-dimensional, stagnation point flow of an incompressible Casson fluid over a porous stretching sheet located at $y=0$. The flow being confined in the region $y>0$. A magnetic field of uniform strength $B_{0}$ is applied perpendicular to the surface. The magnetic Reynolds number is taken to be small enough so that the induced magnetic field can be neglected in comparison to the applied magnetic field.

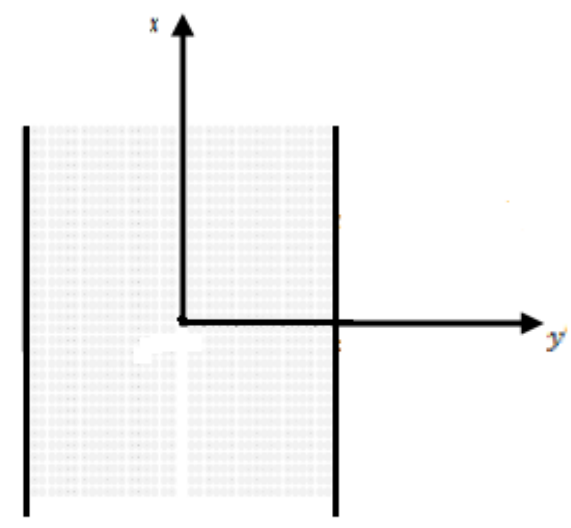

Fig. 1. Geometry of flow

The temperature of the fluid is $T$ and $u, v$ are velocity components. The thermal conductivity and temperature are linearly proportional but other physical quantities are constant.

The stress tensor for an isotropic and incompressible flow of the Casson fluid

$$
\tau_{i j}=\left\{\begin{array}{l}
2\left(\frac{\mu_{B}+p_{y}}{\sqrt{2 \pi}}\right) e_{i j}, \pi>\pi_{c} \\
2\left(\frac{\mu_{B}+p_{y}}{\sqrt{2 \pi_{c}}}\right) e_{i j}, \pi<\pi_{c}
\end{array}\right.
$$


Here, $\tau_{i j}$ is the stress tensor, $e_{i j}$ stand for $(i, j)^{\text {th }}$ component of the deformation rate, $\pi_{c}$ denotes critical value, $p_{y}$ is the yield stress of the fluid. $\mu_{B}$ is plastic dynamic viscosity of the non-Newtonian fluid, and So, if a shear stress less than the yield stress is applied to the fluid, it behaves like a solid, whereas if a shear stress greater than yield stress is applied, it starts to move.

Under the above assumptions, the governing equations of the conservation of mass, momentum, energy in the presence of magnetic field are

$$
\begin{aligned}
& \frac{\partial u}{\partial x}+\frac{\partial v}{\partial y}=0 \\
& u \frac{\partial u}{\partial x}+v \frac{\partial u}{\partial y}=\left(1+\frac{1}{\beta}\right) v \frac{\partial^{2} u}{\partial y^{2}}+g \beta_{T}\left(T-T_{\infty}\right)-\frac{\sigma B_{0}^{2}}{\rho} u-\frac{v}{K_{1}} u \\
& \rho c_{p}\left(u \frac{\partial T}{\partial x}+v \frac{\partial T}{\partial y}\right)=k \frac{\partial^{2} T}{\partial y^{2}}-\frac{\partial q}{\partial y}
\end{aligned}
$$

The appropriate boundary conditions for the velocity components and temperature are given by

$$
\begin{aligned}
& u=u_{w}(x)=c x, v=v_{w}=\text { const }, \quad-k\left(\frac{\partial T}{\partial y}\right)=h\left(T_{f}-T\right) \text { at } y=0 \\
& u \rightarrow 0, T \rightarrow T_{\infty} \text { as } y \rightarrow \infty
\end{aligned}
$$

Using the following similarity variable:

$$
u=c x f^{\prime}(\eta), \quad v=-\sqrt{v c} f(\eta), \eta=\sqrt{\frac{c}{v}} y, \theta(\eta)=\frac{T-T_{\infty}}{T_{f}-T_{\infty}}
$$

Where $\psi=x \sqrt{v c} f(\eta)$ is the stream function with $u=\frac{\partial \psi}{\partial y}$ and $v=-\frac{\partial \psi}{\partial x}$,

The continuity Eq. (2) is automatically satisfied.

Where $c$ is constant, and $T_{w}(x)=T_{\infty}+D x^{\alpha^{*}} \theta(\eta)$ at $\eta=0$ and variable thermal conductivity, $k=k_{\infty}[1+\varepsilon \theta], k_{\infty}$ is the fluid stream conductivity and $\varepsilon$ is defined as $\varepsilon=\frac{\left(k_{w}-k_{\infty}\right)}{k_{\infty}}$, the radiation term $q_{r}=-\frac{4 \sigma^{*}}{3 \beta^{*}} \frac{\partial T^{4}}{\partial y}, \beta^{*}$ is as mean absorption coefficient, $\sigma^{*}$ Stefan Boltzmann constant in the thermal radiation, With the assumption that $T^{4}$ is expanded in Taylor series about $T_{\infty}$ and neglecting higher order terms to get

$$
T^{4} \approx 4 T_{\infty}^{3} T-3 T_{\infty}^{4} \text { and }-\frac{\partial q_{r}}{\partial y}=\frac{4 \sigma^{*} T_{\infty}^{3}}{3 \beta^{*}} \frac{\partial^{2} T}{\partial y^{2}}
$$


Substituting the above appropriate relation, the Eq. (3) to (5) give the following non-linear ordinary differential equations.

$$
\begin{aligned}
& \left(1+\frac{1}{\beta}\right) f^{\prime \prime \prime}+f f^{\prime \prime}-f^{\prime 2}-M f^{\prime}+\lambda \theta-K f^{\prime}=0 \\
& (1+\varepsilon \theta+R) \theta^{\prime \prime}=P_{r}\left[\alpha^{*} \theta f^{\prime}-f \theta^{\prime}\right]
\end{aligned}
$$

The corresponding boundary conditions are

$$
\begin{aligned}
& f(0)=S, f^{\prime}(0)=1, \theta^{\prime}(0)=-\gamma[1-\theta(0)], \quad \text { at } \eta=0 \\
& f^{\prime} \rightarrow 0, \theta \rightarrow 0 \text { as } \eta \rightarrow \infty
\end{aligned}
$$

where $M=\frac{\sigma B_{0}^{2}}{\rho c}$ is magnetic parameter, $\lambda=\frac{G r_{x}}{\operatorname{Re}_{x}^{2}}, G r_{x}=\frac{g \beta\left(T_{f}-T_{\infty}\right) x^{3}}{v^{2}}$ is the local Grashof number, $\operatorname{Re}_{x}=\frac{u_{w} x}{v}$ is the local Renold number, $P_{r}=\frac{\mu c_{p}}{k_{\infty}}$ the Prandtl number, $\gamma=\frac{h}{k} \sqrt{\frac{v}{c}}$ is the Biot number, $\beta=\frac{\mu_{B} \sqrt{2 \pi_{c}}}{p_{y}}$ is Casson fluid parameter, $K=\frac{v}{c K_{1}}$ is the porosity parameter, $S=\frac{-v_{0}}{\sqrt{c v}}$ is the suction/injection parameter, $R=\frac{16 \sigma^{*} T_{\infty}^{3}}{3 \beta^{*} k_{\infty}}$ is the radiation parameter, Roseland mean absorption coefficient is $\beta^{*}$, which measure the ratio of momentum diffusivity to the thermal diffusivity. The prime denotes the differentiation with respect to $\eta$.

\section{Results and Discussion}

The system of coupled equations (8) to (10) is highly non-linear and involves higher order derivatives. These equations are difficult to yield analytical solution. In order to obtain a numerical solution of the problem, the order of the derivatives of these equations is reduced to first order.

We let, $p=f^{\prime}, q=f^{\prime \prime}, g=\theta^{\prime}$,

$$
\begin{aligned}
& q^{\prime}=M p+p^{2}+f q-\lambda \theta+K p \\
& (1+\varepsilon \theta+R) g^{\prime}=P_{r}\left[\alpha^{*} \theta p-f g\right]
\end{aligned}
$$

And the boundary conditions become:

$$
\begin{aligned}
& p(0)=1, g(0)=-\gamma(1-\theta(0)) \\
& p(\infty)=0, \theta(\infty)=0
\end{aligned}
$$


In order to examine the effects of influential parameters for the flow problem, the set of non-linear ordinary differential equations (11 to 13) is solved numerically by using appropriate codes on computational software Mathematica. The physical insight of the problem is revealed through graphs of velocity and temperature functions.

The Figs. 2 and 3 respectively display the effect of suction injection parameter on velocity $f^{\prime}$. It is noticed that velocity decreases in magnitude with increase in suction and opposite behavior is seen for injection. The effect of Prandtl number on velocity $f^{\prime}$ and temperature function $\theta(\eta)$ is demonstrated respectively in the Figs. 4 and 5. The Prandtl number has decreasing effect on both of the physical quantities. The curves for the velocity $f^{\prime}$ and temperature function $\theta(\eta)$ as presented respectively in Figs. 6 and 7, to illustrate the impact of the magnetic field. It is seen that increase in the magnetic field parameter $M$ causes decrease in flow velocity but an increase in the temperature distribution.

Fig. 8 shows that the velocity $f^{\prime}$ is reduced in magnitude with increase in Casson parameter $\beta$.

The curves in the Figs. 9 and 10 respectively indicate the effects of parameter $\lambda$ on function $f^{\prime}$ and $\theta(\eta)$. It is noticed that velocity increases but temperature distribution decreases with increase in the value of $\lambda$ .The increase in the value of the porosity parameter $K$ causes decrease in horizontal velocity $f^{\prime}$ as shown in Fig. 11.

The Figs. 12 and 13 respectively demonstrate the influence of parameter $\varepsilon$ on horizontal velocity $f^{\prime}$ and temperature $\theta(\eta)$. The parameter $\varepsilon$ has small increasing effect on $f^{\prime}$ but it has significant increasing effect on $\theta(\eta)$. Figs. 14 and 15 respectively illustrate the impact of the suction and injection phenomena on temperature distribution. It is observed that $\theta(\eta)$ decrease with increase in suction $(s>0)$ but opposite result is seen for injection $(s<0)$. The influence of Biot number $\gamma$ on temperature function $\theta(\eta)$ is displayed in Fig. 16. The temperature distribution increase in $\gamma$.

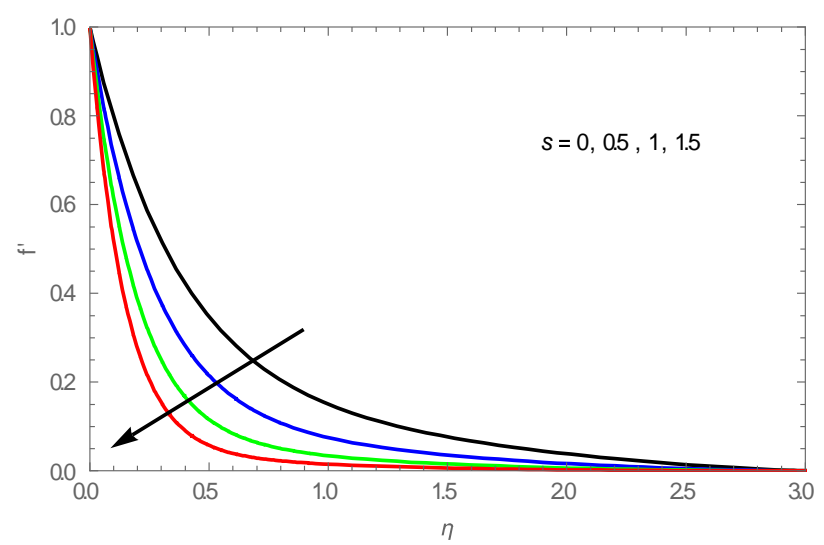

Fig. 2. The plot for curves of $f^{\prime}$ under the suction parameter $s(s>0)$ when

$$
M=0.5, \lambda=0.3, \gamma=1, \beta=1, \mathrm{~K}=0.1 \boldsymbol{P}_{r}=0.7
$$




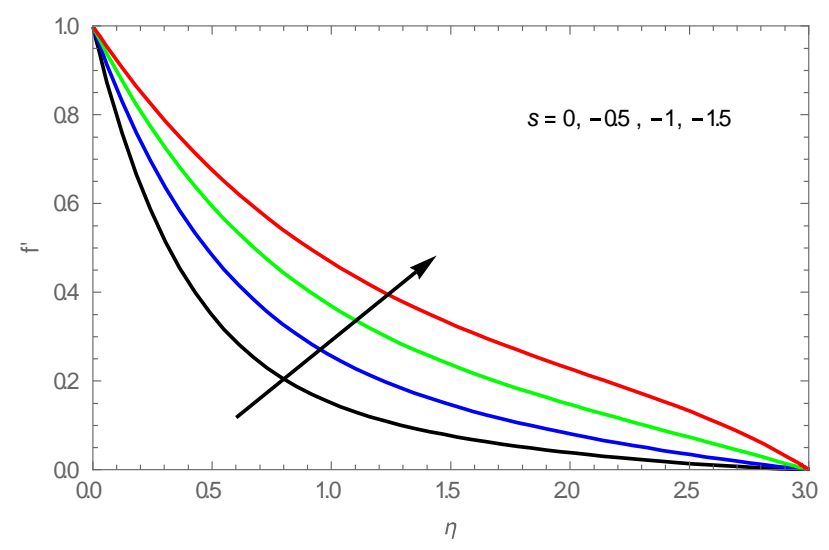

Fig. 3. The plot for curves of $f^{\prime}$ under the injection parameter $s(s<0)$ when $M=0.5, \lambda=0.3, \gamma=1, \beta=1, \mathbf{K}=0.1 \boldsymbol{P}_{r}=0.7$

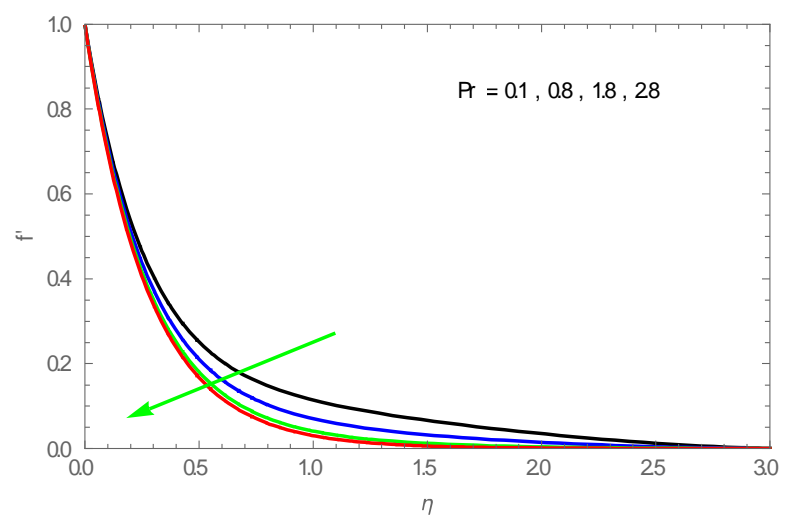

Fig. 4. The plot for curves of $f^{\prime}$ under the effect of Prandtl number when $s=1, M=0.5, \lambda=0.3, \gamma=1, \beta=1, \mathrm{~K}=0.1$

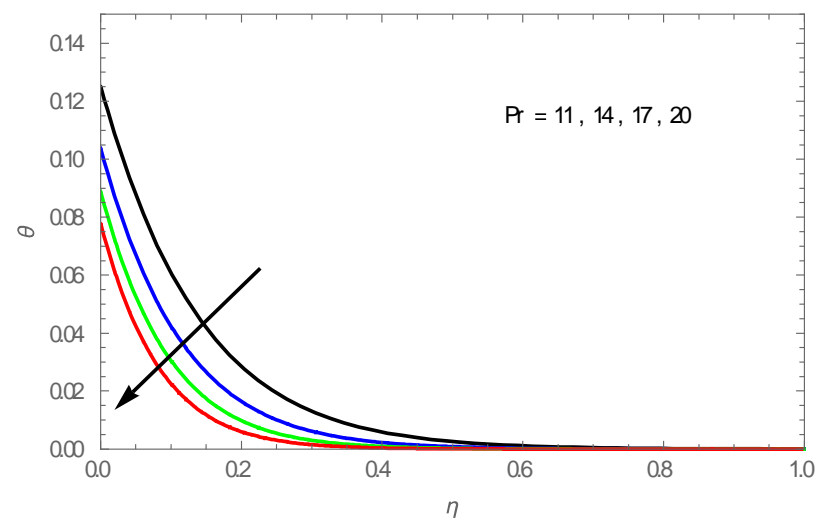

Fig. 5. The plot for curves of $\boldsymbol{\theta}$ under the effect of Prandtl number $\boldsymbol{P}_{\boldsymbol{r}}$ when $s=1, M=0.5, \lambda=0.3, \gamma=1, \beta=1, \mathrm{~K}=0.1$ 


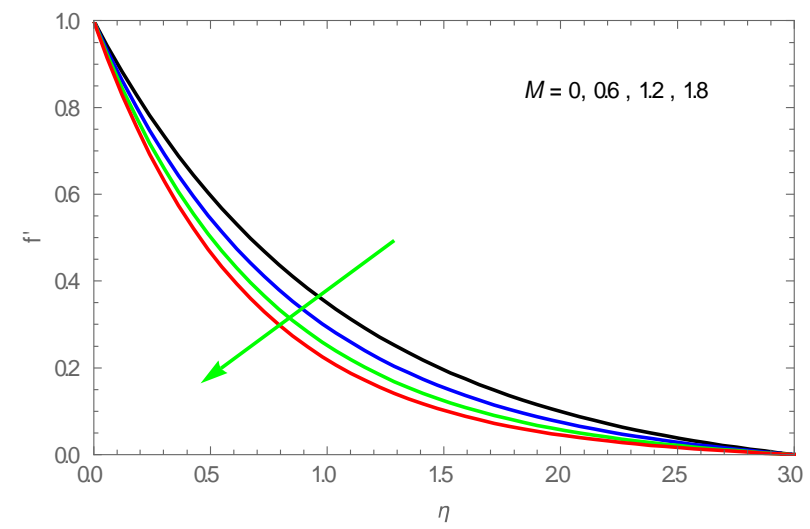

Fig. 6. The plot for curves of $f^{\prime}$ under the magnetic parameter $M$ when $s=1, \lambda=0.3, \gamma=1, \beta=1, \mathrm{~K}=0.1 \boldsymbol{P}_{r}=0.7$

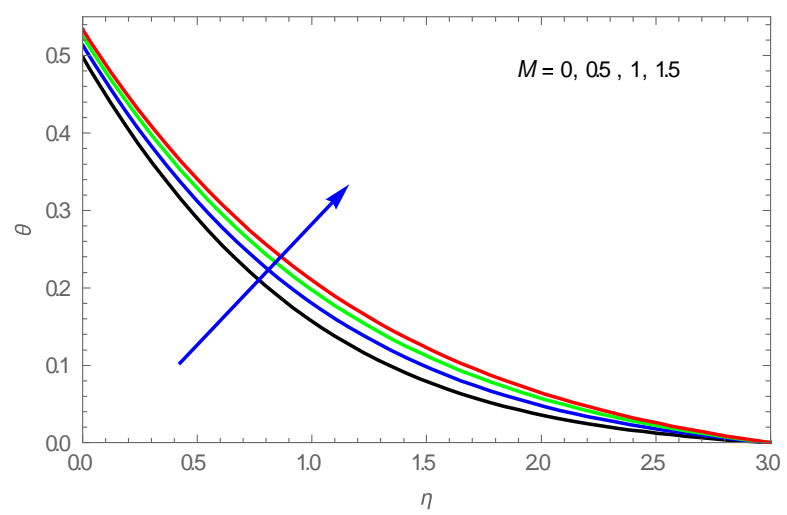

Fig. 7. The plot for curves of $\theta(\eta)$ under the magnetic parameter $M$ when $s=1, \lambda=0.3, \gamma=1, \beta=1, \mathrm{~K}=0.1 \boldsymbol{P}_{r}=0.7$

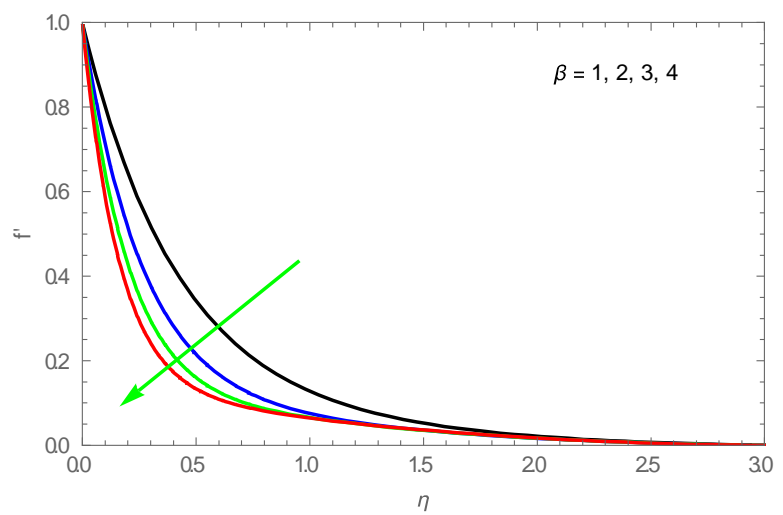

Fig. 8. The plot for curves of $f^{\prime}$ under the effect of Casson parameter $\beta$ when $s=1, M=0.5, \lambda=0.3, \gamma=1, \mathrm{~K}=0.1, \boldsymbol{P}_{r}=0.7$ 


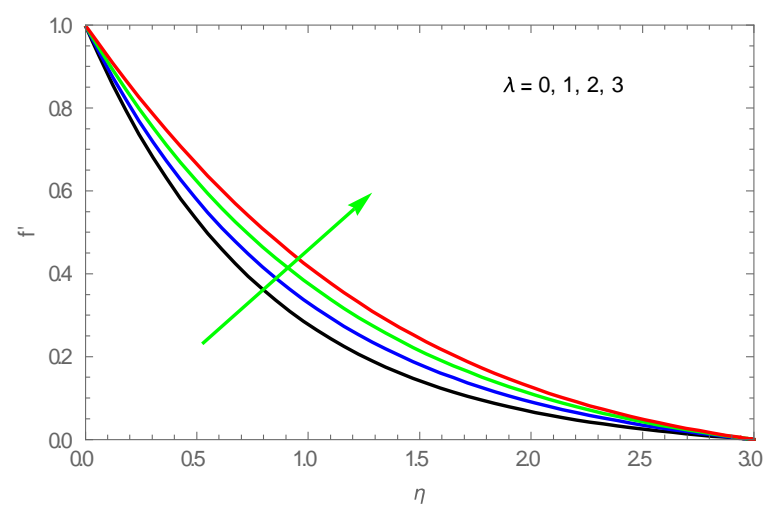

Fig. 9. The plot for curves of $f^{\prime}$ under the effect of parameter $\lambda$ when $s=1, M=0.5, \gamma=1, \beta=1, \mathrm{~K}=0.1 \boldsymbol{P}_{r}=0.7$

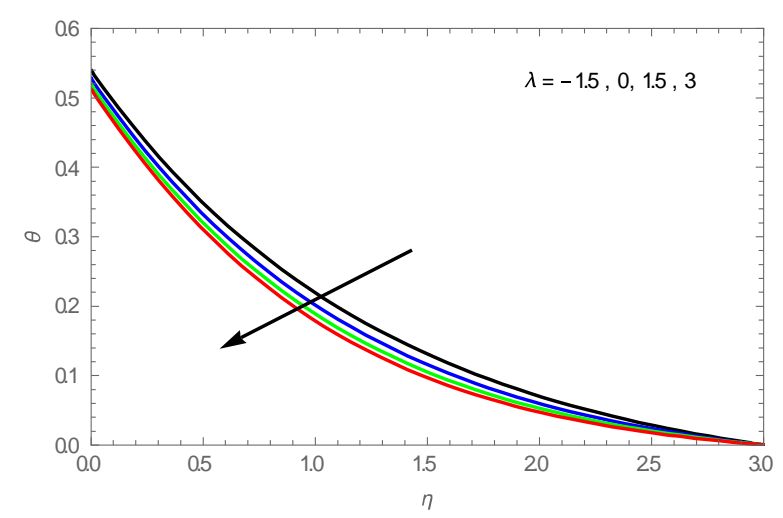

Fig. 10. The plot for curves of $\theta(\eta)$ under the effect of parameter $\lambda$ when $s=1, M=0.5, \gamma=1, \beta=1, \varepsilon=0.2, \mathrm{~K}=0.1 \boldsymbol{P}_{r}=0.7$

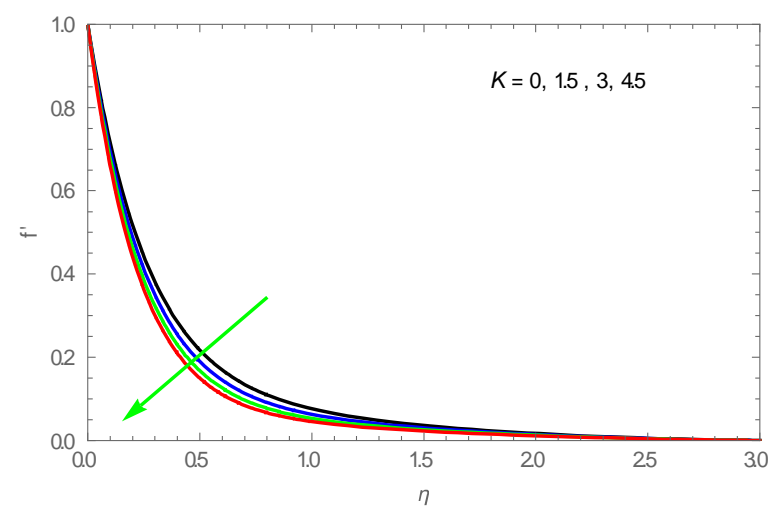

Fig. 11. The plot for curves of $f^{\prime}$ under the effect of porosity parameter $K$ when $s=1, \lambda=0.3, M=0.5, \gamma=1, \beta=1, \varepsilon=0.2, \mathrm{~K}=0.1 \boldsymbol{P}_{r}=0.7$ 


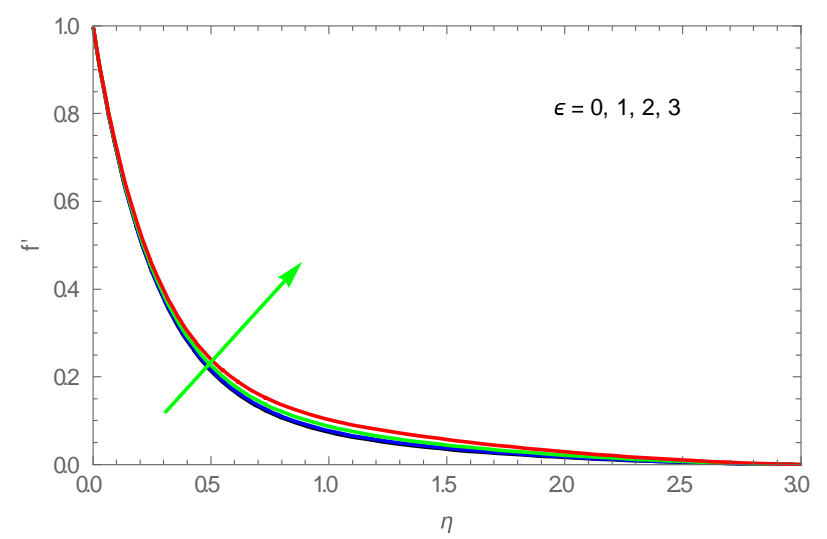

Fig. 12. The plot for curves of $f^{\prime}$ under the parameter $\varepsilon(\varepsilon>0)$ when $s=1, \lambda=0.3, M=0.5, \gamma=1, \beta=1,, \mathrm{~K}=0.1 \boldsymbol{P}_{r}=0.7$

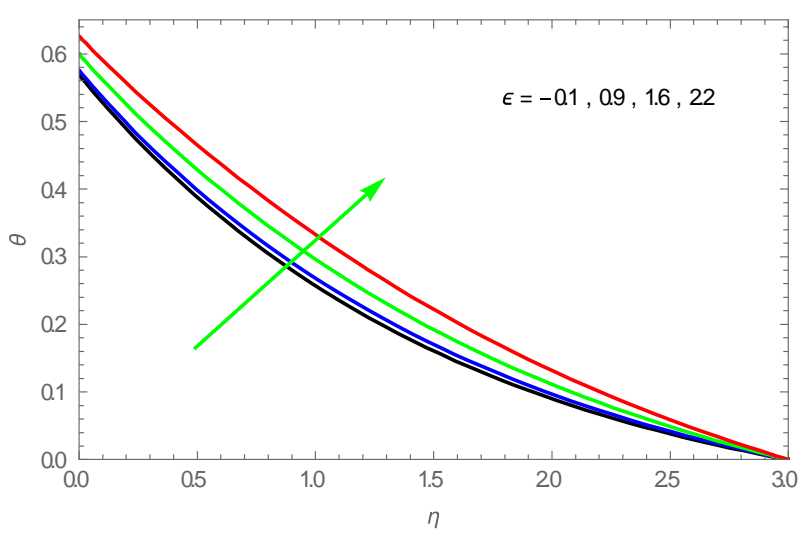

Fig. 13. The plot for curves of $\theta(\eta)$ under the parameter $\varepsilon \quad(\varepsilon<0)$ when $s=1, \lambda=0.3, M=0.5, \gamma=1, \beta=1,, \mathrm{~K}=0.1 \boldsymbol{P}_{r}=0.7$

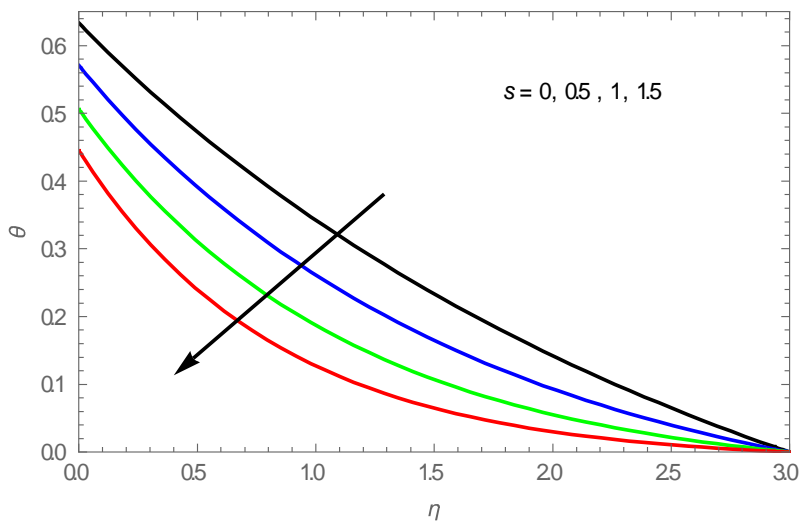

Fig. 14. The plot for curves of $\theta(\eta)$ under the effect of suction parameter $s \quad(s>0)$ when $\lambda=0.3, M=0.5, \gamma=1, \beta=1, \mathcal{E}=0.2, \mathbf{K}=0.1 \boldsymbol{P}_{\boldsymbol{r}}=0.7$ 


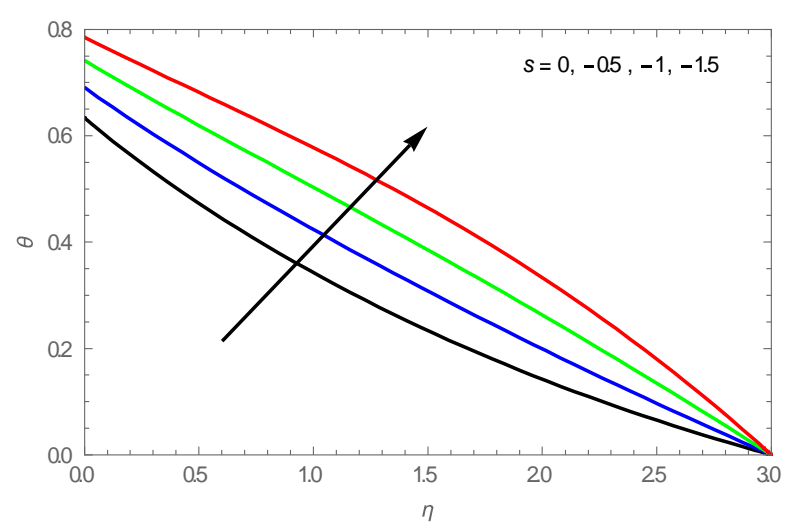

Fig. 15. The plot for curves of $\theta(\eta)$ under the effect of injection parameter $s(s<0)$ when $\lambda=0.3, M=0.5, \gamma=1, \beta=1, \varepsilon=0.2, \mathrm{~K}=0.1 \boldsymbol{P}_{r}=0.7$

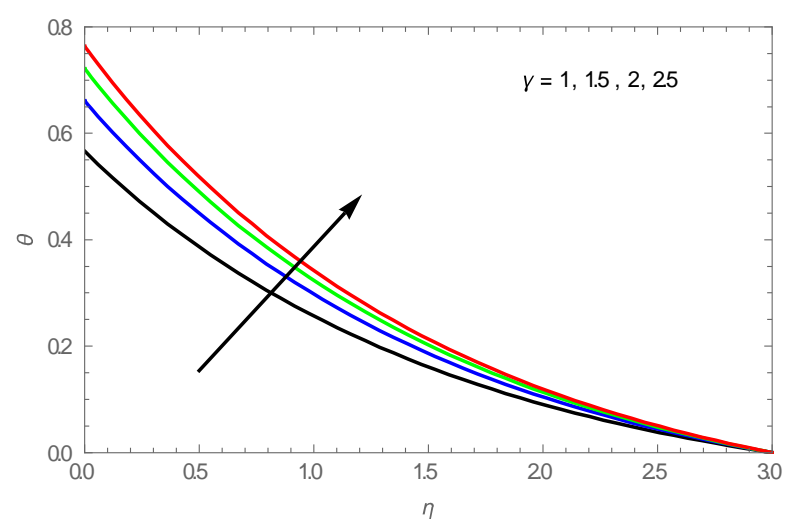

Fig. 16. The plot for curves of $\theta(\eta)$ under the effect of Biot parameter $\gamma$ when $s=1, \lambda=0.3, M=0.5, \beta=1, \varepsilon=0.2, \mathrm{~K}=0.1 \boldsymbol{P}_{r}=0.7$

\section{Conclusion}

The main findings of this work are summarized as follows:

- The horizontal velocity $f^{\prime}$ decreases in magnitude with increase in suction and opposite behavior is seen for injection.

- The Prandtl number has decreasing effect on velocity $f^{\prime}$ and temperature function.

- The magnetic field parameter $M$ causes decrease in flow velocity but an increase in the temperature distribution.

- The velocity $f^{\prime}$ reduces in magnitude with increase in Casson parameter $\beta$.

- The velocity increases but temperature distribution decreases with increase in the value of $\lambda$.

- The increase in the value of the porosity parameter $K$ causes decrease in horizontal velocity $f^{\prime}$.

- The parameter $\mathcal{E}$ has small increasing effect on $f^{\prime}$ but it has significant increasing effect on $\theta(\eta)$. 
- It is observed that $\theta(\eta)$ decrease with increase in suction $(s>0)$ but opposite result is seen for injection $(s<0)$.

- The temperature distribution increase in $\gamma$.

\section{Competing Interests}

Authors have declared that no competing interests exist.

\section{References}

[1] Casson N. A flow equation for pigment oil suspensions of the printing ink type. In: Mill, C.C., Ed., Rheology of Disperse Systems, Pergamon Press, Oxford. 1959;84-102.

[2] Sulochana C, Kishor MK, Sandeep N. Nonlinear thermal radiation and chemical reaction effects on MHD 3D casson fluid flow in porous medium. Chemical and Process Engineering Research. 2015; 37:24-36.

[3] Nadeem S, Haq RU, Lee C, MHD flow of a Casson fluid over an exponentially shrinking sheet. Scientia Iranica. 2012;19:1550-1553.

[4] Raju CSK, Sandeep N, Sugunamma V, Jaya CM, Ramana RJV. Heat and mass transfer in magneto hydrodynamic casson fluid over an exponentially permeable stretching surface. Engineering Science and Technology, an International Journal. 2015;19(1):45-52.

[5] Mustafa M, Hayat T, Pop I, Aziz A. Unsteady boundary layer flow of a Casson fluid due to an impulsively started moving flat plate. Heat Transfer. 2011;40:563-576.

Available:http://dx.doi.org/10.1002/htj.20358

[6] Bhattacharyya K, Hayat T, Alsaedi A. Exact solution for boundary layer low of Casson fluid over a permeable stretching/shrinking sheet. Zeitschrit fur Angewandte Mathematik und Mechanik. 2014;94: 522-528.

Available:http://dx.doi.org/10.1002/zamm.201200031

[7] Bhattacharyya K, Hayat T, Alsaedi A. Analytic solution for magneto hydrodynamic boundary layer low of Casson fluid over a stretching/shrinking sheet with wall mass transfer. Chinese Physics B. 2013;22:Article ID: 024702.

Available:http://dx.doi.org/10.1088/1674-1056/22/2/024702

[8] Nakamura M, Sawada T. Numerical study on the flow of a Non-Newtonian fluid through an axisymmetric stenosis. Journal of Biomechanical Engineering. 1988;110:137-143.

Available:http://dx.doi.org/10.1115/1.3108418

[9] Bird RB, Dai GC, Yarusso BJ. The rheology and flow of viscoplastic materials. Reviews in Chemical Engineering. 1983;1:1-70.

[10] Nadeem S, Haq R, Akbar N, Khan Z. MHD three dimensional Casson fluid flow past a porous linearly stretching sheet. Alex. Eng. J. 2013;52:577-582.

[11] Animasaun I. Effects of thermo pharoses, variable viscosity and thermal conductivity on free convective heat and mass transfer of non-darcian MHD dissipative Casson fluid flow with suction and nth order of chemical reaction. J. Nigerian Math. Soc. 2015;34:11-31. 
[12] Nadeem S, Mehmood R, Akbar N. Optimized analytical solution for oblique flow of a Casson-nano fluid with convective boundary conditions. Int. J. Therm. Sci. 2014;78:90-100.

[13] Akbar N, Khan Z. Metachronal beating of cilia under the influence of Casson fluid and magnetic field. J. Magn. Magn. Mater. 2015;378:320-326.

[14] Akbar N, Ebaid A, Khan Z. Numerical analysis of magnetic field effects on Eyring-Powell fluid flow towards a stretching sheet. J. Magn. Magn. Mater. 2015;382:355-358.

[15] Akbar N. Influence of magnetic field on peristaltic flow of a Casson fluid in an asymmetric channel: application in crude oil refinement. J. Magn. Magn. Mater. 2015;378:463-468.

[16] Benazir AJ, Sivraj R, Makind OD. Unsteady MHD Casson fluid flow over a vertical cone and flat plate with non-uniform heat source/sink. Int. J. Eng. Res. Africa. 2015;21:69-83.

[17] Hassan W, Sajjad H, Shamila K. Chemaical diffusion and thermal effects on MHD stagnation oint flow of Casson fluids past a poous surface. International Journal of Scientific and Engineering Research. 2017;8(3):1092-1103.

[18] Hassan W, Rafique S, Khalid S, Ahamad F, Hussain S. Thermal radiation effects on unsteady MHD flow of Casson fluids through porous medium over a permeable shrinking sheet. J. Appl. Environ. Biol. Sci. 2017;7(4):201-209.

[19] Nadeem T, Sajjad H. Magnetohydrodynamic flow of Casson fluids over a moving boundary surface. J. Appl. Environ. Biol. Sci. 2017;7(4):192-200,

[20] Nadeem AS, Farhad A, Muhammad S, Ilyas K, Syed A. A comparative study of Atangana-Baleanu and Caputo-Fabrizio fractional derivatives to the convective flow of a generalized Casson fluid. The European Physical Journal Plus. 2017;132:54.

[21] Nadeem AS, Farhad A, Muhammad S, Ilyas K, Syed AAli SA, Metib SA. Comparison and analysis of the Atangana-Baleanu and Caputo-Fabrizio fractional derivatives for generalized Casson fluid model with heat generation and chemical reaction. Results in Physics. 2017;7:789-800.

[22] Farhad A, Nadeem AS, Ilyas K, Muhammad S. Magnetic field effect on blood flow of Casson fluid in axisymmetric cylindrical tube: A fractional model. Journal of Magnetism and Magnetic Materials. 2017;327-336.

[23] Yamamoto K, Iwamura N. Flow with convective acceleration through a porous medium. J Phy Soc Jpn. 1976;37(3):41.

[24] Mounuddin K, Pattabhiramacharyulu NCh. Steady flow of a viscous fluid through a saturated porous medium of finite thickness, impermeable and thermally insulated bottom and the other side is stress free, at a constant temperature. J Pure Appl Phys. 2010;22(1):107-22.

[25] Chamkha AJ. MHD free convection from a vertical plate embedded in a thermally stratified porous medium. Fluid/Part Separ J. 1996;9(3):195-206.

[26] Makinde OD, Aziz A. MHD mixed convection from a vertical plate embedded in a porous medium with a convective boundary condition. Int J Therm Sci. 2010;49:1813-20.

[27] Makinde OD, Mhone PY. On temporal stability analysis for hydromagnetic flow in a channel filled with a saturated porous medium. Flow Turbul Combust. 2009;83:21-32. 
[28] Makinde OD. On MHD boundary-layer flow and mass transfer past a vertical plate in a porous medium with constant heat flux. Int J Num Meth Heat Fluid Flow. 2009;19(3/4):546-54.

[29] Hayat T, Shehzad A, Muhammad Q, Alsaedi A. Mixed convection flow by a porous sheet with variable thermal conductivity and convective boundary condition. Brazilian Journal of Chemical Engineering. 2014;31(01):109 - 117.

(c) 2017 Waqas et al.; This is an Open Access article distributed under the terms of the Creative Commons Attribution License (http://creativecommons.org/licenses/by/4.0), which permits unrestricted use, distribution, and reproduction in any medium, provided the original work is properly cited.

Peer-review history:

The peer review history for this paper can be accessed here (Please copy paste the total link in your

browser address bar)

http://sciencedomain.org/review-history/19614 